\title{
Diffuse Cosmic Rays Shining in the Galactic Center: A Novel Interpretation of H.E.S.S. and Fermi-LAT $\gamma$-Ray Data
}

\begin{abstract}
D. Gaggero, ${ }^{1, *}$ D. Grasso, ${ }^{2, \dagger}$ A. Marinelli, ${ }^{2, \$}$ M. Taoso, ${ }^{3,8}$ and A. Urbano ${ }^{4, \|}$
${ }^{1}$ GRAPPA, University of Amsterdam, Science Park 904, 1098 XH Amsterdam, Netherlands

${ }^{2}$ INFN Pisa and Pisa University, Largo B. Pontecorvo 3, I-56127 Pisa, Italy

${ }^{3}$ Instituto de Física Teórica (IFT), UAM/CSIC, Cantoblanco, 28049 Madrid, Spain

${ }^{4}$ CERN, Theoretical Physics Department, 1211 Geneva, Switzerland

(Received 10 February 2017; revised manuscript received 26 April 2017; published 17 July 2017)

We present a novel interpretation of the $\gamma$-ray diffuse emission measured by Fermi-LAT and H.E.S.S. in the Galactic center (GC) region and the Galactic ridge (GR). In the first part we perform a data-driven analysis based on PASS8 Fermi-LAT data: We extend down to a few $\mathrm{GeV}$ the spectra measured by H.E.S.S. and infer the primary cosmic-ray (CR) radial distribution between 0.1 and $3 \mathrm{TeV}$. In the second part we adopt a CR transport model based on a position-dependent diffusion coefficient. Such behavior reproduces the radial dependence of the CR spectral index recently inferred from the Fermi-LAT observations. We find that the bulk of the GR emission can be naturally explained by the interaction of the diffuse steady-state Galactic CR sea with the gas present in the central molecular zone. Although we confirm the presence of a residual radial-dependent emission associated with a central source, the relevance of the large-scale diffuse component prevents to claim a solid evidence of GC pevatrons.
\end{abstract}

DOI: 10.1103/PhysRevLett.119.031101

Introduction.-The High Energy Stereoscopic System (H.E.S.S.) Collaboration recently reported the discovery of a $\gamma$-ray diffuse emission from a small region surrounding SgrA* [1]. The emission spectrum is compatible with a single power law with index $\Gamma_{\text {HESS16 }}=2.32 \pm 0.05_{\text {stat }} \pm$ $0.11_{\text {syst }}$ and extends up to $\sim 50 \mathrm{TeV}$ with no statistically significant evidence of a cutoff. If hadronic, as expected due to the strong losses suffered by electrons in that region, that emission may point to the presence of a proton population with energies up to the $\mathrm{PeV}$ in the Galactic center (GC).

On the basis of the angular profile of the emission, the H.E.S.S. Collaboration proposed the J1745-290 source as its possible origin. This source is positionally compatible with the SgrA* supermassive black hole and with the G 359.95-0.04 pulsar wind nebula. Although the observed spectrum of HESS J1745-290 is suppressed above $\sim 10 \mathrm{TeV}$, this might be explained by the attenuation due to the presence of a dense radiation field around that source (see, e.g., Ref. [2]). Annihilating dark matter in the halo central spike [3], or a peaked population of cosmic rays (CRs) interacting with high concentrated gas in that region, could also explain the diffuse emission measured by H.E.S.S. The H.E.S.S. results have raised wide interest as it seems to provide the first evidence of pevatrons in our Galaxy.

Published by the American Physical Society under the terms of the Creative Commons Attribution 4.0 International license. Further distribution of this work must maintain attribution to the author(s) and the published article's title, journal citation, and DOI.
A $\gamma$-ray diffuse emission was also measured by a previous H.E.S.S. observational campaign towards the so-called Galactic ridge (GR) [4]. That emission approximately traces the gas distribution in the central molecular zone (CMZ) - a massive structure rich in molecular gas that extends up to $\sim 250$ pc away from the GC along the Galactic plane (GP). Its spectrum is compatible with a single power law with index $\Gamma_{\text {HESS06 }}=2.29 \pm 0.07_{\text {stat }} \pm 0.20_{\text {syst }}$, which, although observed only up to $\sim 10 \mathrm{TeV}$, is in agreement with that found in the inner region surrounding $\operatorname{SgrA}^{*}$.

The spectra of the CR population that one can infer from these data are significantly harder than the local $\mathrm{CR}$ spectrum measured at the Earth position $\left[\Gamma_{\mathrm{CR}}\left(r_{\odot}\right) \simeq 2.7\right.$ for $E_{\mathrm{CR}}>300 \mathrm{GeV} /$ nucleon see, e.g., Refs. [5,6]]. On the other hand, at lower energies, Fermi-LAT observations of the SgrB complex in the CMZ suggest a CR spectrum similar to the local one [7].

The H.E.S.S. Collaboration proposed that the discrepancy could be the signature of a freshly accelerated CR population, possibly originated by SgrA* or by other sources in the central parsec of the Galaxy.

The aim of this Letter is to estimate the contribution of the CR large-scale population (hereafter the CR sea) to the diffuse emission measured by H.E.S.S. and Fermi-LAT in the GC region, and to provide a consistent interpretation of those data. Differently from previous computations, we model the CR sea by relaxing the simplified hypothesis of a uniform spectral index in the Galaxy. This approach is motivated by recent analyses of Fermi-LAT data [8-10] showing that the $\gamma$-ray diffuse emission of the Galaxy, and hence the CR primary spectrum, gets harder approaching the GC along the GP. 


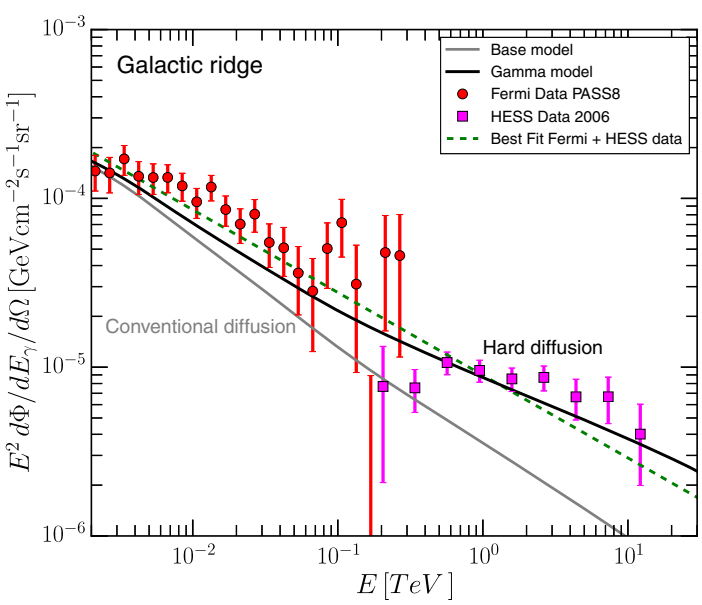

FIG. 1. The $\gamma$-ray spectrum in the GR region $\left(|l|<0.8^{\circ}\right.$, $|b|<0.3^{\circ}$ ). Fermi-LAT data, shown here for the first time, and H.E.S.S. data from Ref. [4] are compared with the contribution of the Galactic CR sea as computed with the gamma and base models discussed in the text. The single power-law best fit of the combined data is also reported. We have subtracted the contribution of point sources from Fermi-LAT data.

In Ref. [8] this behavior was interpreted in terms of a radial dependence of both the scaling of the CR diffusion coefficient with rigidity, and the advection velocity. The phenomenological model based on these ingredients reproduces the Fermi-LAT data in most of the regions of the sky, including the inner GP where conventional models provide an unsatisfactory fit [11]. Later, it was shown [12] that the same scenario is in agreement with the high-energy data as well, providing a viable solution to the long-standing Milagro anomaly, i.e., an excess of the diffuse emission in the inner GP at $15 \mathrm{TeV}$ with respect to the predictions of conventional models [13]. Moreover, this setting may also imply a significant Galactic contribution to the astrophysical neutrino flux recently measured by IceCube [12] (see also Ref. [14]).

Here we adopt the same scenario and, using a detailed 3D gas model for the CMZ region [15], compute the contribution of the CR sea to the $\gamma$-ray diffuse emission from the GC region. We compare our results with 2006 and 2016 H.E.S.S. data and, for the first time in this context, with Fermi-LAT PASS 8 data for the same region. We will show (see Figs. 1 and 2) that-above $10 \mathrm{GeV}$-this contribution is significantly larger and harder than the one estimated so far on the basis of conventional models. Therefore, we propose that a large fraction of the $\gamma$-ray emission measured by H.E.S.S. and Fermi-LAT near the GC and in the whole GR is originated by the diffuse, steady-state Galactic CR sea interacting with the massive molecular clouds in the CMZ.

Fermi-LAT data analysis.-The Fermi-LAT Collaboration recently released a new set of data based on the PASS8 event reconstruction algorithm [16]. In comparison to previous analyses, this approach yields a larger

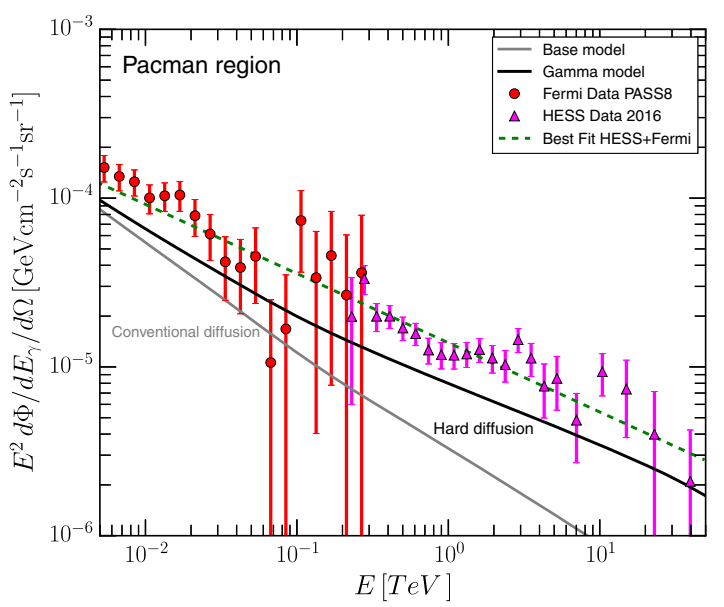

FIG. 2. Same as for Fig. 1 but for the pacman region defined in the text.

effective area, hence more statistics for the same observation time, as well as better energy and angular resolutions. Such improved performances are valuable in this context since they allow us to improve the morphological and spectral information of the emission in the small portion of the sky under scrutiny.

We extract Fermi-LAT data using the Fermi Science TOOLS V10R0P5 [17]. We use 422 weeks of PASS 8 data with the event class CLEAN and we apply the recommended quality cuts: (DATA_QUAL $==1) \& \&\left(L A T \_C O N F I G==1\right)$. Moreover events with zenith angles larger than $90^{\circ}$ are excluded. The exposure is computed using the Fermi-LAT response function P8REP2 CLEAN V6. The data are binned in 30 energy bins equally spaced in $\log$ scale between $300 \mathrm{MeV}$ and $300 \mathrm{GeV}$. The counts and the exposure maps have been produced using the HEALPIX pixelization scheme [18], with a resolution $n_{\text {side }}=1024$, corresponding to a pixel size of $\sim 0.06^{\circ}$.

The emission from the point sources is obtained from the 4-year Point Source Catalog (3FGL) provided by the Fermi-LAT Collaboration [19]. We also considered the high-energy 2FHL catalog finding only one source in the considered sky window, which is compatible with 3FGL J1745.6-2859c at the GC. We model the point source emission convolving the flux of each 3FGL source with the point spread function (PSF) of the instrument, which is derived using the GTPSF Fermi tool.

In Figs. 1 and 2 we report the Fermi-LAT and H.E.S.S. observations in the GR $\left(|l|<0.8^{\circ},|b|<0.3^{\circ}\right)$ and in the region considered in Ref. [1], an open annulus centered on SgrA* with $\theta_{\text {inner }}=0.15^{\circ}$ and $\theta_{\text {outer }}=0.45^{\circ}$, (hereafter called the "pacman"). The improved statistics provided by the PASS 8 algorithm allows, for the first time, an overlap between Fermi-LAT and H.E.S.S. data around $200 \mathrm{GeV}$, covering therefore the entire energy range between $0.3 \mathrm{GeV}$ and $50 \mathrm{TeV}$. Noticeably, the two data sets are consistent with a single power law both in the GR and the pacman regions: The $95 \%$ C.L. single-power law fits from $10 \mathrm{GeV}$ to $10 \mathrm{TeV}$ in the two regions are, respectively, 
$\Phi_{\mathrm{GR}}=8.96_{-1.39}^{+1.35} \times 10^{-9}\left(\frac{E_{\gamma}}{1 \mathrm{TeV}}\right)^{-2.49_{-0.08}^{+0.09}}\left(\mathrm{TeV} \mathrm{cm}^{2} \mathrm{~s} \mathrm{sr}\right)^{-1}$

and

$\Phi_{\mathrm{pm}}=1.36_{-0.12}^{+0.12} \times 10^{-8}\left(\frac{E_{\gamma}}{1 \mathrm{TeV}}\right)^{-2.41_{-0.06}^{+0.07}}\left(\mathrm{TeV} \mathrm{cm}^{2} \mathrm{~s} \mathrm{sr}\right)^{-1}$

with reduced $\chi^{2}=3$, and 1.4.

We find only mild changes of our results using the Fermi event type PSF3, which corresponds to a subset of the events with a better angular reconstruction.

In the rest of this section we use the angular dependence of the diffuse emission measured by Fermi-LAT to infer the CR energy density radial profile $w_{\mathrm{CR}}(r)$ in the CMZ region, for energies corresponding to $E_{\mathrm{CR}} \geq 100 \mathrm{GeV}$. We will then compare its shape with that determined by the H.E.S.S. Collaboration for $E_{\mathrm{CR}}>10 \mathrm{TeV}$ [1]. Possible discrepancies among those profiles may reveal the presence of a nonstationary CR source since charged particles with different energies diffuse with different time scales. Moreover, Fermi-LAT data extend to larger longitudes than H.E.S.S., which may allow us to better probe the large radii tail of the $\mathrm{CR}$ distribution.

For consistency, we determine $w_{\mathrm{CR}}$ using the same expression adopted in Ref. [1] [Eq. (2) in the Supplemental Material of that paper] correcting it to account for the energy dependence of the pion production cross section. This gives

$$
\begin{aligned}
& w_{\mathrm{CR}}\left(E_{\mathrm{CR}} \geq 0.1 \mathrm{TeV}\right) \\
& \quad=3.9 \times 10^{-2} \mathrm{eV} \mathrm{cm}^{-3} \\
& \quad \times\left(\frac{\eta_{N}}{1.5}\right)^{-1}\left(\frac{L_{\gamma}(\geq 10 \mathrm{GeV})}{10^{34} \mathrm{erg} / \mathrm{s}}\right)\left(\frac{M_{\mathrm{gas}}}{10^{6} M_{\odot}}\right)^{-1} .
\end{aligned}
$$

Here $L_{\gamma}\left(\geq E_{\gamma}\right)$ is the $\gamma$-ray luminosity above $E_{\gamma}$ in each region (subtracting the contribution from point sources); $M_{\text {gas }}$ is the corresponding total hydrogen mass; $\eta_{N} \approx 1.5$ is a factor accounting for the presence of heavier nuclei.

Using the Fermi tools we extract the diffuse luminosity $L_{\gamma}\left(E_{\gamma} \geq 10 \mathrm{GeV}\right)$ in an annulus and in six adjacent circular regions with angular diameter of $0.2^{\circ}$ centered on the plane intersecting SgrA* (see Fig. 3). These regions are larger than those considered by H.E.S.S., which is motivated by the smaller angular resolution of Fermi-LAT. To determine the gas mass distribution we use the same CS column density map [23] adopted by the H.E.S.S. Collaboration [1].

The resulting CR energy density radial profile $w_{\mathrm{CR}}(r)$ in the energy range $0.1 \leq E_{\mathrm{CR}} \leq 0.3 \mathrm{TeV}$ is reported in Fig. 3, as well as the CR distribution derived by the H.E.S.S. Collaboration in Ref. [1] for $E_{\mathrm{CR}} \geq 10 \mathrm{TeV}$. Although the large errors and scatter of the points based

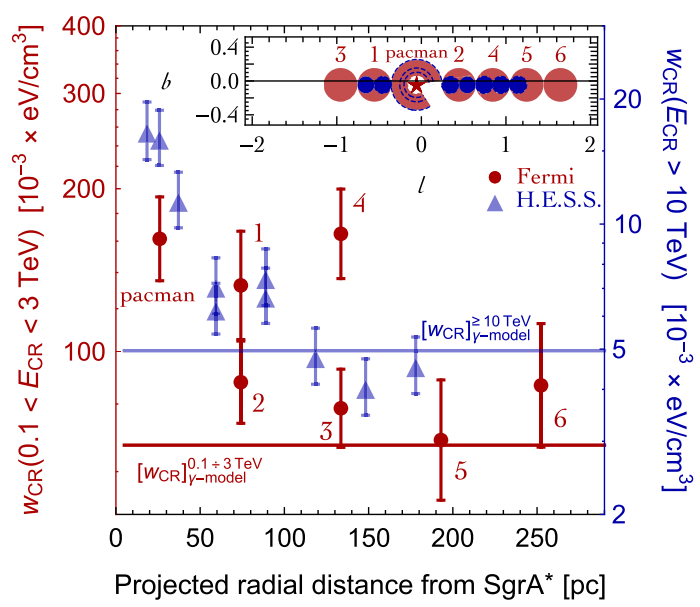

FIG. 3. The CR energy density radial profiles for $E_{\mathrm{CR}}>10 \mathrm{TeV}$, as determined by H.E.S.S. [1], and for $0.1 \leq E_{\mathrm{CR}} \leq 3 \mathrm{TeV}$, as determined here from Fermi-LAT data, are reported. Those data are compared with the gamma model predictions (solid lines). The regions of the sky used for deriving the data are represented in the inset. The model energy density profiles on Galactic scales are reported in the Supplemental Material [20].

on Fermi-LAT data do not allow a tight constraint at low energies, our results are consistent with an energy independent shape of the CR density profile. It is clear that both data sets are consistent with being constant for $r \gtrsim 100$ pc.

Phenomenological model.-In this section we compare the previous results with the phenomenological scenario proposed in Ref. [8]. This model was designed to reproduce the $\gamma$-ray spectra in the inner GP measured by Fermi-LAT, which were found to be harder than those predicted by conventional models [11]. The scenario, which was implemented in the DRAGON code $[24,25]$, assumes that the exponent $\delta$, setting the scaling of the $\mathrm{CR}$ diffusion coefficient with rigidity, has a linear dependence on the galactocentric radius $(r): \delta(r)=A r+B$. The parameters $A$ and $B$ were tuned to consistently reproduce CR and Fermi-LAT $\gamma$-ray data on the whole sky. In particular, the so-called $\mathrm{KRA}_{\gamma}$ model adopts $A=0.035 \mathrm{kpc}^{-1}$ and $B=0.21$, giving $\delta\left(r_{\odot}\right) \simeq 0.5$. Assuming a uniform CR source spectral index across the whole Galaxy, this behavior turns into a radial dependence of the propagated CR spectral index, producing longitudedependent $\gamma$-ray spectra along the GP. Remarkably, this is in reasonably good agreement with the results of a recent Fermi-LAT analysis [9] (see Fig. 8 in that paper) as well as with those reported in Ref. [10] on the basis of the same data (Note, however, that in Ref. [10] the CR spectrum at the GC is slightly softer than that found by the Fermi-LAT Collaboration.) A radial dependence of the advection velocity was also adopted in Ref. [8]. Advection, however, plays no relevant role in the energy range considered in this work.

Similar to Ref. [12], here we introduce a spectral hardening in the proton and Helium source spectra at $\sim 300 \mathrm{GeV} / \mathrm{n}$, in order to reproduce the local propagated 
spectra measured by PAMELA [5], AMS-02 [6], and CREAM [26]. We assume this feature to be present in the whole Galaxy, as it may be expected if it is produced by propagation effects. Under these conditions, the KRA $\gamma$ model was shown [12] to reproduce the emission observed by Milagro in inner GP at a $15 \mathrm{TeV}$ median energy [13] consistent with Fermi-LAT data. At larger energies we assume a cutoff to be present in the CR source spectra at $\sim 5 \mathrm{PeV} /$ nucleon, so as to match KASCADE-Grande results [27], though this feature has no effect in the energy interval considered in this Letter.

We compute the $\pi^{0}$, Inverse-Compton, and bremsstrahlung components of the $\gamma$-ray diffuse emission, integrating the convolution of the spatially dependent CR spectrum, gas or radiation density distributions and proper cross sections along the line of sight. The $\pi^{0}$ component is dominant in the GC region. We checked that, for reasonable choices of the interstellar radiation field (ISRF), the $\gamma$-ray opacity in the $\mathrm{CMZ}$ region is negligible in the energy range considered in this work. Here we adopt the ISRF taken from the latest public version of GALPROP [28,29] and pion production cross sections as parametrized in Ref. [30]. The scenario proposed in Ref. [8] predicts a CR proton spectral index $\Gamma_{\mathrm{CR}}(r \simeq 0)=\Gamma_{\mathrm{CR}}\left(r_{\odot}\right)-A r_{\odot}$. Then, since $\Gamma_{\mathrm{CR}}\left(r_{\odot}\right) \simeq 2.7$ above $\sim 300 \mathrm{GeV}$, this implies $\Gamma_{\mathrm{CR}}(r \simeq 0) \simeq 2.4$, in agreement with that found in the previous section. We notice that this finding is independent on the value of the parameter $B$ setting the normalization of $\delta$ so that tuning this quantity to better match recent AMS-02 B/C results [31] would not affect our results.

With respect to what was reported in Ref. [8], here we replace the hydrogen distribution in the inner $3 \mathrm{kpc}$ with the three-dimensional analytical model presented in Ref. [15], as required to properly model the hadronic emission in that region. Outside that region we adopt the gas model used in Refs. [28,29]. The main components are molecular $\left(\mathrm{H}_{2}\right)$ and atomic $(\mathrm{HI})$ and hydrogen. $\mathrm{HI}$, which is inferred from $21-\mathrm{cm}$ lines, is less than $10 \%$ of the total mass. $\mathrm{H}_{2}$ is not observed directly; except for the densest clumps, the column density can be inferred from several tracers, most commonly from the $\mathrm{CO}$ emission lines. This requires a conversion factor that was estimated to be $X_{\mathrm{CO}}(r \sim 0) \simeq 0.5 \times 10^{20} \mathrm{~cm}^{-2} \mathrm{~K}^{-1} \mathrm{~km}^{-1} \mathrm{~s}$ with a factor 2 uncertainty [15]. Here we use $X_{\mathrm{CO}}(r \sim 0) \simeq$ $0.6 \times 10^{20} \mathrm{~cm}^{-2} \mathrm{~K}^{-1} \mathrm{~km}^{-1} \mathrm{~s}$, the value giving the best agreement with the integrated mass distribution, based on the CS emission map, reported in Ref. [1]. The quoted uncertainty on this parameter directly applies to the diffuse $\gamma$-ray emission normalization. This effect, however, is degenerate with that of varying the CR (poorly known) source density at the GC.

Following Ref. [8], we use the CR source distribution of Ref. [21], based on supernova remnant catalogs. This parametrization vanishes at the GC, a behavior in qualitative agreement with the $\gamma$-ray emissivity profile determined by the Fermi-LAT Collaboration [9], which displays a dip in the
GC. For a given transport model, this choice minimizes the $\mathrm{CR}$ sea density in the CMZ region. We verified that using the source distribution reported in Ref. [22], which does not vanish at the $\mathrm{GC}$, turns into a factor 2 larger emission from the GR and pacman regions. This is still compatible with experimental data. Moreover, this enhancement can be compensated by a reduction of the $X_{\mathrm{CO}}$ factor leaving it well within the observationally allowed range.

In Figs. 1 and 2 we show the gamma-ray emission in the GR and pacman regions predicted by this model (hereafter the gamma model). For comparison we also report the spectrum computed for a conventional model (base model), sharing with the gamma model all the properties, but keeping the diffusion coefficient and the convective velocity spatially uniform (The main parameters characterizing the base and gamma models are reported in Table 1 in the Supplemental Material [20].) We find that the base (as any other conventional) model cannot consistently reproduce the H.E.S.S. and Fermi-LAT measurements in the absence of an additional component with a harder spectrum. Instead, in the GR the gamma model is in excellent agreement with the shape and normalization of the measured spectrum.

In the pacman region the gamma model prediction lies slightly below the data. This is consistent with what is inferred from the CR energy density radial profile $w_{\mathrm{CR}}$, shown in Fig. 3, which in respect to the CR sea (almost uniform on those small scales) displays a peak toward the GC. We interpret this feature to be due to one (or more) CR source(s) in the inner few pc of the Galaxy. Far outside that region $(r \gtrsim 100 \mathrm{pc})$, we find that the CR energy density in both energy ranges $0.1 \leq E_{\mathrm{CR}} \leq 0.3 \mathrm{TeV}$ and $E_{\mathrm{CR}} \geq 10 \mathrm{TeV}$, is in good agreement with experimental data.

Although not shown here, we have also checked that the gamma model is in excellent agreement with the Fermi-LAT and H.E.S.S. observations in the SgrB complex region $(0.4<l<0.9,-0.3<l<0.2)$.

Conclusions. - In this Letter we have shown that a large fraction of the $\gamma$-ray emission from the CMZ measured by H.E.S.S. and Fermi-LAT from few $\mathrm{GeV}$ up to $50 \mathrm{TeV}$ can be originated by the same population of energetic particles. In fact, we have found that the Galactic CR sea accounts for the bulk of that emission if it is modeled under the assumption of a spatial-dependent diffusion. This feature is motivated by the radial dependence of the CR spectral index recently inferred from Fermi-LAT data. Therefore, our results provide a new strong evidence supporting the validity of that scenario in a region of the Galaxy were the discrepancies between the base and conventional model are expected to be maximal.

On top of this diffuse emission, we have outlined-by means of an energy-dependent data-driven analysis - the hint for the presence of a larger CR density in the vicinity of the central radio source Sgr A* with respect to the average density inferred from the whole GR, similarly to that that found by H.E.S.S. at larger energy. We have not found any 
significant evidence of a different spectral shape between those regions.

Therefore, this excess may be originated by one or more CR sources in the inner few parsecs of the Galaxy, which are likely to be responsible for the J1745-290 emission. No firm conclusion, however, can be drawn on the maximal energy of $\mathrm{CR}$ accelerated by these sources since the significance of the $\gamma$-ray excess in the pacman region with respect to the contribution of the CR sea is rather small above $10 \mathrm{TeV}$.

In the future, the South site of the CTA [32] may provide a further confirmation of the scenario discussed in this Letter from the detailed observation of a larger region centered on the GC.

We warmly thank L. Baldini, C. Evoli, S. Gabici, M. Pesce-Rollins, and P. Ullio for valuable discussions. We also thank A. Viana for providing us the CS column density map used by the H.E.S.S. Collaboration. M. T. is supported by the Centro de Excelencia Severo Ochoa Programme SEV-2012-0249, MINECO through a Severo Ochoa fellowship with the Program SEV-2012-0249, FPA2015-65929-P, and Consolider MultiDark CSD200900064. Pac-Man was designed by T. Iwatani.

Note added in proof.-Recently, the H.E.S.S. Collaboration published a new analysis of the $\gamma$-ray emission observed in the inner $200 \mathrm{pc}$ of the Galaxy based on 250 hours of data [33]. The spectrum of that emission extends up to $45 \mathrm{TeV}$ and is compatible with that observed in the pacman region. That result is consistent with the interpretation proposed in this Letter.

*D.Gaggero@uva.nl

†dario.grasso@pi.infn.it

*antonio.marinelli@pi.infn.it

§m.taoso@csic.es

alfredo.leonardo.urbano@cern.ch

[1] A. Abramowski et al. (H.E.S.S. Collaboration), Nature (London) 531, 476 (2016).

[2] S. Celli, A. Palladino, and F. Vissani, Eur. Phys. J. C 77, 66 (2017).

[3] T. Lacroix, J. Silk, E. Moulin, and C. Bœ hm, Phys. Rev. D 94, 123008 (2016).

[4] F. Aharonian et al. (H.E.S.S. Collaboration), Nature (London) 439, 695 (2006).

[5] O. Adriani et al. (PAMELA Collaboration), Science 332, 69 (2011).

[6] M. Aguilar et al. (AMS Collaboration), Phys. Rev. Lett. 114, 171103 (2015).

[7] R. Yang, D. Jones, and F. Aharonian, Astron. Astrophys. 580, A90 (2015).

[8] D. Gaggero, A. Urbano, M. Valli, and P. Ullio, Phys. Rev. D 91, 083012 (2015).

[9] F. Acero et al. (Fermi-LAT Collaboration), Astrophys. J. Suppl. Ser. 223, 26 (2016).
[10] R. Yang, F. Aharonian, and C. Evoli, Phys. Rev. D 93, 123007 (2016).

[11] M. Ackermann et al. (Fermi-LAT Collaboration), Astrophys. J. 750, 3 (2012).

[12] D. Gaggero, D. Grasso, A. Marinelli, A. Urbano, and M. Valli, Astrophys. J. 815, L25 (2015).

[13] A. A. Abdo et al., Astrophys. J. 688, 1078 (2008).

[14] G. Pagliaroli, C. Evoli, and F. L. Villante, J. Cosmol. Astropart. Phys. 11 (2016) 004.

[15] K. Ferriere, W. Gillard, and P. Jean, Astron. Astrophys. 467, 611 (2007).

[16] W. Atwood et al. (Fermi-LAT Collaboration), arXiv: 1303.3514.

[17] M. Ackermann et al. (Fermi-LAT Collaboration), Astrophys. J. Suppl. Ser. 203, 4 (2012).

[18] K. M. Gorski, E. Hivon, A. J. Banday, B. D. Wandelt, F. K. Hansen, M. Reinecke, and M. Bartelman, Astrophys. J. 622, 759 (2005).

[19] F. Acero et al. (Fermi-LAT Collaboration), Astrophys. J. Suppl. Ser. 218, 23 (2015).

[20] See Supplemental Material at http://link.aps.org/ supplemental/10.1103/PhysRevLett.119.031101 for (i) In the table "Parameters of our reference propagation models" we report the main properties/parameters which characterize the two cosmic-rays propagation setups represented in our work. (ii) The plot represents the large scale cosmic ray energy density radial profile in the energy interval $0.1<E<3 \mathrm{TeV}$ for the gamma (continuos lines) and base (dashed lines) models and for two different radial distribution of sources: Case and Bhattacharya (Ref. [21]) and Yusifov and Küçük (Ref. [22]).

[21] G. L. Case and D. Bhattacharya, Astrophys. J. 504, 761 (1998).

[22] I. Yusifov and I. Küçük, Astron. Astrophys. 422, 545 (2004).

[23] M. Tsuboi, T. Handa, and N. Ukita, Astrophys. J. 120, 1 (1999).

[24] C. Evoli, D. Gaggero, D. Grasso, and L. Maccione, J. Cosmol. Astropart. Phys. 10 (2008) 018; 04 (2016) E01.

[25] C. Evoli, D. Gaggero, A. Vittino, G. Di Bernardo, M. Di Mauro, A. Ligorini, P. Ullio, and D. Grasso, J. Cosmol. Astropart. Phys. 02 (2017) 015.

[26] H. Ahn, P. Allison, M. Bagliesi, J. Beatty, G. Bigongiari et al., Astrophys. J. 714, L89 (2010).

[27] W. Apel et al., Astropart. Phys. 47, 54 (2013).

[28] The galprop code for cosmic-ray transport and diffuse emission production, http://galprop.stanford.edu/.

[29] A. E. Vladimirov, S. W. Digel, G. Johannesson, P. F. Michelson, I. V. Moskalenko, P. L. Nolan, E. Orlando, T. A. Porter, and A. W. Strong, Comput. Phys. Commun. 182, 1156 (2011).

[30] T. Kamae, N. Karlsson, T. Mizuno, T. Abe, and T. Koi, Astrophys. J. 647, 692 (2006); 662, 779E (2007).

[31] M. Aguilar et al. (AMS Collaboration), Phys. Rev. Lett. 117, 231102 (2016).

[32] B. S. Acharya, M. Actis, T. Aghajani, G. Agnetta, J. Aguilar, F. Aharonian, M. Ajello, A. Akhperjanian, M. Alcubierre, J. Aleksić et al., Astropart. Phys. 43, 3 (2013).

[33] H. Abdalla et al. (HESS Collaboration), arXiv:1706.04535. 